\title{
Analysis of Influencing Factors and Predictive Models of Multidrug-resistant Bacterial Infection in Severe Patients
}

Xianhui Wang

Jilin Central Hospital, Jilin City 132000, Jilin Province, China

issues all over the world. Patients in the intensive care unit (ICU) have more opportunities for pathogen invasion, with immunosuppressive agents and complex underlying diseases. The risk of infection by multidrug-resistant bacteria in ICU is much higher than that in other departments. In order to cope with the emergence of antibiotic resistance, ICU doctors should follow the principle of minimizing unnecessary use of antibiotics in accordance with antibiotic management guidelines. However, studies have shown that most patients in China's ICU are still using antibiotics, and more than half of them are infected with multi-drug resistant bacteria ${ }^{[1]}$. In addition, due to the long detective time for multidrugresistant bacteria, how to balance antibiotic infection and multidrug-resistant bacteria is an important challenge for ICU physicians. This study aims to analyze the risk factors of multidrug resistance in critically ill patients and establish a predictive model.

\section{Information and methods}

\subsection{Information}

207 infected patients in ICU from 2018 to May 2020 were selected, of which 73 were infected by multidrug resistant bacteria. Among them, the patients were between 21 and 73 years old, with an average age of 44.8 .

\subsection{Methods}

\subsubsection{Drug sensitivity test}

Collecting, separating, nurturing, and identifying infected samples were conducted according to inspection procedures. The results of bacterial identification and drug susceptibility testing were 
used to analyze multi-drug resistant bacterial infections in hospitals.

\subsubsection{Evaluation criteria}

Multi-drug-resistant bacterial infections, strains that infect 3 or more pathogenic bacteria against antibacterial drugs.

\subsection{Statistical methods}

SPSS20.0 statistical software was used for analysis. The count data are expressed in terms of the number of cases and the composition ratio, using $\mathrm{X} 2$ test.

\section{Results}

Table 1. Screening of risk factors of multi-drug resistant bacterial infection

\begin{tabular}{|c|c|c|}
\hline Risk factor & $\begin{array}{c}\text { Non-multidrug-resistant } \\
\text { bacterial infections }(n=134)\end{array}$ & $\begin{array}{c}\text { Multiple drug-resistant } \\
\text { bacterial infections }(n=73)\end{array}$ \\
\hline \multicolumn{3}{|l|}{ Basic information } \\
\hline Age(year) & $58.43 \pm 16.29$ & $65.50 \pm 13.46$ \\
\hline Hospital time(d) & $2.74 \pm 15.61$ & $17.87 \pm 5.1$ \\
\hline ICU time(d) & $9.00 \pm 9.70$ & $16.59 \pm 5.13$ \\
\hline Fever(d) & $0.00 \pm 0.00$ & $7.13 \pm 25.18$ \\
\hline \multicolumn{3}{|l|}{ Times of abnormality in lab testing } \\
\hline Routine analysis of blood & $24.54 \pm 18.90$ & $44.11 \pm 23.41$ \\
\hline Procalcitonin & $6.39 \pm 4.86$ & $13.18 \pm 7.36$ \\
\hline Routine test of urine & $6.07 \pm 6.48$ & 15.0519 .40 \\
\hline \multicolumn{3}{|l|}{ Use of anti-bacteria drugs } \\
\hline Day of using anti-bacteria drugs(d) & $18.19 \pm 11.61$ & $25.39 \pm 14.02$ \\
\hline \multicolumn{3}{|l|}{ Use of anti-bacteria drugs after surgery } \\
\hline Yes & $83(59.7)$ & $43(53.8)$ \\
\hline No & $56(40.3)$ & $37(46.2)$ \\
\hline Days of using anti-bacteria drugs after surgery(d) & $7.93 \pm 9.32$ & $13.78 \pm 16.12$ \\
\hline \multicolumn{3}{|l|}{ Position of infection[ 例 $(\%)]$} \\
\hline Ventilation system & $82(59.0)$ & $46(57.5)$ \\
\hline Cardio system & $0(0.0)$ & $1(1.2)$ \\
\hline Blood system & $11(7.9)$ & $8(10.0)$ \\
\hline Abdomen and digestive system & $7(5.0)$ & $8(10.0)$ \\
\hline Urinary system & $30(216)$ & $13(163)$ \\
\hline Skin and soft tissue & $3(2.2)$ & $1(1.2)$ \\
\hline Genital tract & $1(0.7)$ & $0(0.0)$ \\
\hline Others & $5(3.6)$ & $3(3.8)$ \\
\hline
\end{tabular}

\subsection{Forecasting model}

observation of multi-drug resistance is close

\subsection{Analysis of risk factors in patients with multi-} drug resistant bacteria infection

Incorporating the information of 207 patients into the statistical analysis, 134 patients were infected by non-multidrug-resistant bacteria, and 73 patients were infected by multidrug-resistant bacteria. Infection by multidrug-resistant bacteria was accounted for $35.27 \%$ of all infected patients. Single-factor screening was performed on the risk factors of the multi-drug resistant bacteria infection group and the non-multidrug resistant bacteria infection group (Table 1).

The relevance between forecasting model and actual 


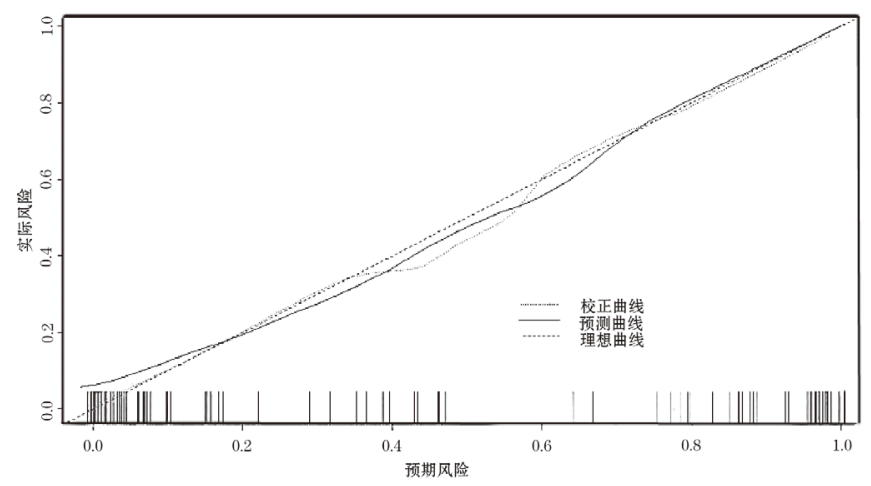

Figure 1. Curve graph of multi-drug resistance

\section{Discussion}

The rapid development of advanced medical technology, the widespread application of invasive surgery, the unreasonable and frequent use of antibiotics, and the irregular treatment of chronic inflammation have led to a significant increase of multi-drug resistant bacterial infections in hospital ${ }^{[1]}$. MDRO refers to bacteria that are resistant to three or more antibiotics at the same time, which can significantly increase the length of hospitalization, treatment costs and mortality. Bacterial resistance has attracted the attention of scholars at home and abroad, and bacterial resistance has become the main problem to cope with in the field of infection. The National Health and Family Planning Commission clearly requested the hospital infection control department to treat methicillin-resistant Staphylococcus aureus (MRSA), vancomycin-resistant enterococcus (VRE), broad-spectrum-lactamase (ESBL) and other multidrugs resistance bacteria. And hospital should control enterobacteria (CRE) resistant to carbapenems ${ }^{[2]}$.

As a statistical graph forecasting tool, graphs can provide more intuitive forecasting results. At the same time, multidrug-resistant bacterial infections are difficult to treat and easily cause disease outbreaks, which are the main factors leading to increased medical expenses and mortality. Currently, due to the multidrug resistance of antimicrobial agents, this problem has become more and more prominent ${ }^{[3]}$. This study is an exploratory study based on single factor analysis which is used to visually identify the risk factors for multidrug-resistant bacterial infections in the ICU. The key technical problem to be solved is the transition from analyzing a single infected patient to analyzing the interaction between risk factors. Risk factors for multidrug-resistant bacterial infections are average length of hospital stay, days of fever, abnormal laboratory tests, antibiotic use, days of ventilation, urinary catheter use, and emergency surgery. Further analysis showed that the multi-drug resistant bacterial infection group has a higher risk factor network density, and the mutual influence between risk factors is closer ${ }^{[4]}$. The number of fever days, the abnormal number of procalcitonin, and the number of days after surgery to use antibacterial drugs are the key factors in the risk factor network and the key factors in the interaction of multiple risk factors, so caution should be taken. In addition, in this study, methicillin-resistant Staphylococcus aureus is sensitive to both teicoplanin and vancomycin. No drug-resistant strains have been found so far, and no Staphylococcus aureus has been found resistant to vancomycin in domestic studies in the last two years ${ }^{[5]}$. Antimicrobial agents with higher resistance rates include erythromycin, clindamycin, levofloxacin, tetracycline and gentamicin, and the detection rate of methicillin-resistant Staphylococcus aureus is higher among Staphylococcus aureus ${ }^{[6]}$.

In addition to the above statistical analysis, the causes of multi-drug resistant bacterial infections include: (1) ICU patients have low immune function and poor anti-infection ability. Newborns and elderly patients living in the intensive care unit are prone to hospital infections due to weakened immune function and decreased anti-infection ability. The use of corticosteroids, cyclophosphamide and azathioprine and other immunosuppressants alone or in combination can suppress various levels of immunity in the body, reduce the patient's ability to resist infection, and lay the foundation for the occurrence of infection. Oncology drugs or radiation therapy for malignant tumors can suppress the bone marrow and reduce the white blood cell count. The body's immune system is damaged and is prone to nosocomial infection ${ }^{[7]}$.

(2) The contamination of the environment by pathogenic bacteria in the intensive care unit. Intensive care patients in the intensive care unit have more intensive care equipment and various advanced treatment equipment. Therefore, more and more medical staff use invasive surgery, which has higher mobility and takes up less space. ICU personnel and patients have a high rate of bacterial infection ${ }^{[8]}$. If the ward is not treated in time, the internal air and surface disinfection of various instruments and equipment 
can easily be infected with various pathogens through contact and transmission.

(3) ICU patients who have undergone various invasive operations usually undergo interventional treatments on the surface of these catheters, such as intravenous infusion tubes, central venous pressure tubes, internal catheters, gastrointestinal decompression tubes, urethral catheters, bronchostomy, and others pathogenic bacteria. When the catheter is inserted into the body, pathogenic bacteria will also enter the body and cause catheter-related infections, such as catheter-related pneumonia, catheter-related bloodstream infection and catheter-related urinary tract infection. Some patients developed ventilator-associated pneumonia after using a ventilator to assist breathing ${ }^{[9]}$.

(4) Widespread application of antibiotics. All patients in ICU wards are critically ill, and the use of antibiotics is very frequent, which greatly promotes the production of resistant bacteria. The bacterium exhibits multi-drug resistance to antibiotics and panantibacterial, which not only brings great difficulties to treatment, but also threatens the safety of patients.

(5) The impact of hand hygiene on pathogenic bacteria of hospital infection. In the process of diagnosis, treatment and nursing in the intensive care unit, medical staff must touch the secretions, blood, body fluids and used items of the patient or the patient, and carry a large number of pathogenic bacteria on their hands. Failure to strictly comply with hand hygiene standards and maintain hand hygiene can lead to cross-infection between doctors and patients ${ }^{[10]}$.

(6) Irregular operation of aseptic technique. When performing intravenous infusion, central venous catheter insertion, catheter placement, tracheostomy, bronchostomy, etc., it may cause iatrogenic infection if you do not follow the aseptic technique operation procedures.

(7) Ill implementation of isolation measures. ICU patients often develop multi-drug resistant bacteria infection or colonization. If the patients who infected or colonized by the same kind of multi-drug resistant bacteria are not isolated in time, the multi-drug resistant bacteria will spread in the ICU ward and an outbreak of multi-drug resistant bacteria infection will occur.

(8) The facilities and layout of the ICU are not even. The infrastructure and layout of the ICU ward are unreasonable and defective, resulting in abnormal or incorrect medical operation procedures. It is unable to effectively control the transmission path, and unable to reach the health indicators of medical work. The ICU ward is a key department of intensive care and treatment, and it is also the location of serious hospital infections. Most patients are severely infected after surgery, and some patients have serious underlying diseases such as tumors, diabetes, heart failure, and kidney failure. They also have a lot of antibiotics, hormones and other invasive procedures.

Therefore, the construction arrangements and facilities of the intensive care unit should be reasonable, the work process should be standardized, and the intensive care unit should be monitored by targets. The relevant factors of hospital infection should be discovered in time to achieve early prevention, isolation and control of hospital infection. In order to monitor multi-drug-resistant bacteria, detect and isolate infections and accumulate multidrug-resistant bacteria in time, and reduce the occurrence of infections, hands should be cleaned before and after diagnosis and treatment, and before direct contact with the patient's body fluids. We should disinfected the ward and use disposable medical supplies. Various items used by patients should be treated in a special way, and curtains are hang to split the ward to form different bed units.

However, other important reasons for pathogen cross-infection are: the relative mobility of air has increased; medical staff's hand washing is not up to standard; aseptic operation is not strict; and frequent home visits are also frequent. Therefore, it is necessary to strengthen the flame-retardant management system and the monitoring of various pathogens in the intensive care unit, strengthen the compliance with the hand washing of medical staff, strictly implement the disinfection and quarantine system, comply with the regulations of aseptic technology, enhance environmental sanitation management, and perform daily inspections on materials and equipment surface. Only in this way can the pathogenic bacterial infection in the intensive care unit be effectively controlled, and the occurrence of infection in the hospital can be effectively prevented and controlled.

Therefore, improper placement of intensive care unit facilities and buildings is an important factor leading to hospital infections. Hospitals should 
strengthen prevention and control measures against drug-resistant bacteria, using antibacterial drugs in accordance with medication requirements. They should strictly implement ward disinfection and quarantine measures and standardize hand hygiene of medical staff. Thereby it can reduce hospital infection rates. At present, the infection of multi-drug resistant bacteria has become a key issue of general concern in many hospitals. All medical institutions must strictly abide by aseptic technique operating procedures. The hospital should strictly implement aseptic technique operating procedures and standard operating procedures to prevent contamination and effectively prevent multidrug-resistant bacterial infections, especially when performing various invasive procedures.

\section{Reference}

[1] Guo WL. The effect of using PDCA cycle to reduce the detection rate of nosocomial infection of multi-drug resistant bacteria[J]. Journal of Clinical Rational Use, 2020, 13(33): 131-133.

[2] Shi DM. Application effect of clustered nursing care of multidrug resistant bacteria infection in geriatric ward[J]. Medical Theory and Practice, 2020, 33(22): 3841-3842.

[3] Cai YY. Application of microbiological examination in hospital infection control[J]. Contemporary Medicine, 2020, 26(33): 123-125.
[4] Chen KF, Yang JK, Zhang M, et al. Clinical analysis and drug resistance monitoring of multi-drug resistant bacteria infection in Leshan area[J]. Practical Preventive Medicine, 2020, 27(11): 1329-1333.

[5] Chen X, Chen LN, Han PP, et al. Analysis of risk factors for multi-drug resistant bacteria infection in patients with ventilator-associated pneumonia in stroke in ICU[J]. Nursing Practice and Research, 2020, 17(21): 7-9.

[6] Li J, Wang YX. The application of PDCA circulation model in the management of multidrug-resistant bacterial infections in critically ill patients in neurosurgery[J]. Nursing Practice and Research, 2020, 17(20): 63-65.

[7] Wang YP, Li NY, Yuan HH, et al. Study on the infection characteristics of multi-drug resistant bacteria in medical institutions in Pudong New Area[J]. Chinese Journal of Disinfection, 2020, 37(10): 733-736+740.

[8] Zhu LJ, Chen SZ, Lin C. Isolation intervention to control the colonization of multi-drug resistant bacteria in intensive care unit[J]. Chinese Journal of Disinfection, 2020, 37(10): 744747.

[9] Mao QY, Jia C, Zhang L, et al. Application research of multidisciplinary cooperation in the prevention and control of multi-drug resistant bacteria infection[J]. Journal of Nursing Management, 2020, 20(10): 749-752.

[10] He G. Application of targeted nursing in the control of multidrug-resistant bacterial infections in intensive care units[J]. Contemporary Nurses (Mid-day Journal), 2020, 27(10): 131132. 